\title{
Correction: High throughput approaches reveal splicing of primary microRNA transcripts and tissue specific expression of mature microRNAs in Vitis vinifera
}

Erica Mica ${ }^{1,2+}$, Viviana Piccolo ${ }^{1 \dagger}$, Massimo Delledonne $^{3}$, Alberto Ferrarini $^{3}$, Mario Pezzotti $^{4}$, Cesare Casati ${ }^{1}$, Cristian Del Fabbro ${ }^{5}$, Giorgio Valle ${ }^{6}$, Alberto Policriti ${ }^{7}$, Michele Morgante ${ }^{5,7}$, Graziano Pesole ${ }^{8,9}$, M Enrico Pè2, David S Horner ${ }^{1 *}$

\begin{abstract}
The version of this article published in BMC Genomics 2009, 10:558, contains data in Table 1 which are now known to be unreliable, and an illustration, in Figure 1, of unusual miRNA processing events predicted by these unreliable data. In this full-length correction, new data replace those found to be unreliable, leading to a more straightforward interpretation without altering the principle conclusions of the study. Table 1 and associated methods have been corrected, Figure 1 deleted, supplementary file 1 added, and modifications made to the sections "Deep sequencing of small RNAs from grapevine leaf tissue" and "Microarray analysis of miRNA expression". The editors and authors regret the inconvenience caused to readers by premature publication of the original paper.
\end{abstract}

Background: MicroRNAs are short ( 21 base) single stranded RNAs that, in plants, are generally coded by specific genes and cleaved specifically from hairpin precursors. MicroRNAs are critical for the regulation of multiple developmental, stress related and other physiological processes in plants. The recent annotation of the genome of the grapevine (Vitis vinifera L.) allowed the identification of many putative conserved microRNA precursors, grouped into multiple gene families.

Results: Here we use oligonucleotide arrays to provide the first indication that many of these microRNAs show differential expression patterns between tissues and during the maturation of fruit in the grapevine. Furthermore we demonstrate that whole transcriptome sequencing and deep-sequencing of small RNA fractions can be used both to identify which microRNA precursors are expressed in different tissues and to estimate genomic coordinates and patterns of splicing and alternative splicing for many primary miRNA transcripts.

Conclusions: Our results show that many microRNAs are differentially expressed in different tissues and during fruit maturation in the grapevine. Furthermore, the demonstration that whole transcriptome sequencing can be used to identify candidate splicing events and approximate primary microRNA transcript coordinates represents a significant step towards the large-scale elucidation of mechanisms regulating the expression of microRNAs at the transcriptional and post-transcriptional levels.

\footnotetext{
* Correspondence: david.horner@unimi.it

† Contributed equally

${ }^{1}$ Dipartimento di Scienze Biomolecolari e Biotecnologie, Università degli

Studi di Milano, Milano, Italy
} 


\section{Background}

This article has been published as a correction for [1].

MicroRNAs (miRNAs) are small (19-24 nt) non-coding RNAs that play important roles in the regulation of various cellular processes by inhibiting gene expression at the post-transcriptional level [2-4]. Many miRNAs interact with target mRNAs, leading to degradation or sequestration from the translational apparatus $[5,6]$; some miRNAs target other non-coding transcripts and are required for the generation of trans-acting small interfering RNAs (ta-siRNAs) [7]. miRNAs have been implicated in the regulation of key developmental, stress response and other physiological processes. While in animals many miRNAs are derived from introns or untranslated regions of coding messages, plant miRNAs are typically specified by dedicated MIR genes. These genes are, at least for the most part, transcribed by RNA polymerase II to yield capped and polyadenylated primary transcripts (pri-miRNA) [8]. The RNAse III enzyme Dicer-like-1 (DCL1) mediates the specific excision of mature miRNAs from the pri-miRNA via the initial generation of imperfect hairpin precursors (premiRNAs) and the subsequent excision of a duplex consisting of the mature microRNA and its complementary region $\left(\right.$ miRNA $\left.^{*}\right)[9,10]$. Most, if not all plant miRNAs then undergo methylation of the 2' hydroxyl group at the 3' ends of this duplex [11] and are subsequently exported to the cytosol, where one strand, the mature miRNA, is selectively incorporated into the RNA Induced Silencing Complex (RISC) which mediates interactions with target mRNAs [12].

The same, or highly similar mature miRNAs are often specified by different genomic loci within a species, and many, but by no means all, miRNAs show broad phylogenetic conservation - similar miRNAs are grouped into families. Several computational methods have been developed to identify putative pre-miRNAs by evaluating the capacity of the genomic context of sequences similar to known mature miRNAs to form hairpin structures exhibiting structural and thermodynamic features consistent with known pre-miRNAs (e.g. [13]). Purely ab-initio approaches to the prediction of non-conserved (lineagespecific) miRNAs have also yielded some notable successes (e.g. [14]), although such approaches are often plagued by an excess of false positive results. The most reliable method to identify putative novel pre-miRNAs remains the sequencing of small RNA fractions [11,15-21] coupled with the identification of plausible hairpin structures in flanking genomic sequences (e.g. [22]).

Once mature miRNA sequences have been identified, their expression in different tissues, developmental stages or environmental conditions can be studied through experimental approaches such as northern blotting, oligonucleotide arrays or deep-sequencing of isolated small RNA fractions. However, the fact that identical or highly similar mature miRNAs can derive from multiple loci within a single genome limits the capacity of such approaches to determine which genomic precursor loci are truly expressed.

Meaningful insight into the regulation of miRNA expression at the transcriptional and other levels is undoubtedly desirable in the context of post-genomic and systems biology initiatives. However, large scale and insilico studies of the regulation of transcription of miRNAs require accurate definition of primary transcript coordinates on a genomic sequence, or at the very least, fairly accurate estimates of transcriptional start sites. Owing to their typically short physiological half-life, relatively few pri-miRNAs have been found in EST and large-scale fulllength cDNA sequencing projects in plants. For the same reasons, manual cloning and characterization of primary miRNAs is a laborious process, and has been performed for relatively few plant miRNAs [8,23-25].

To date, large-scale analysis of expression of plant miRNAs and associated prediction of precursor sequences have been restricted to relatively few species and the paucity of complete plant genome sequences limits the possibilities for studies incorporating extensive genomic information. The recently published genome sequence of the grapevine (Vitis vinifera L.) [26] provides the first opportunity to study the potential roles of miRNAs in fruit maturation and other physiological processes of a commercially important species in the context of a complete genome sequence.

We have previously used comparative methods to predict 140 putative pre-miRNAs (representing 28 conserved miRNA families) in the grapevine genome [26]. Here we present experimental validation of a large number of these predictions using several high throughput methodologies. Oligonucleotide arrays reveal that several of these families show significant changes in expression levels in different tissues and during fruit maturation. Small RNA deep-sequencing allowed the precise definition of boundaries of mature miRNA sequences where comparative predictions left some ambiguity. Additionally, we demonstrate that deep sequencing of the poly ${ }^{+}$ transcriptome permits the precise identification of which candidate precursors are expressed in different tissues and shows that, in many cases, fairly precise estimates of primary transcript coordinates may be inferred from such data. Finally, we show that patterns of splicing and alternative splicing of pri-miRNAs may be elucidated from whole transcriptome deep sequencing data and confirm that a significant proportion of grapevine pri-miRNAs are subjected to such processes, consistent with the suggestion that post-transcriptional regulation 
might play a widespread role in the regulation of plant miRNA maturation.

\section{Results and Discussion}

In the previous description of 8.4 fold coverage assembly of the grapevine (Vitis vinifera L.) genome [26] we identified 164 candidate conserved miRNAs. Here we present a comprehensive characterization of conserved miRNAs in grapevine including a refinement of mature miRNA sequences and important information regarding pattern of expression of both mature miRNAs and precursors. Three complementary approaches were followed to characterize the expression pattern of both the mature miRNAs and their precursors. To allow a comparison between methods, all technologies were applied to leaf tissue. Whole transcriptome deep sequencing was performed on all tissues available from the highly homozygote sequenced clone PN40024. To maximize the coverage of tissues studied, microarray and 454 transcriptomic analyses were performed on berries organs of particular agronomic importance - from other clones as they cannot be easily obtained from the very weak PN40024 clone.

\section{Comparative prediction of microRNA precursors}

We previously used the MicroHarvester software [13] with all plant miRNAs present in release 9.1 of miRBase [27] to identify 164 candidate conserved miRNAs and their precursors in the 8.4 fold coverage assembly of the genome of the grapevine (Vitis vinifera L.) [26]. Manual refinement of these predictions provided 140 high-confidence candidate pre-miRNAs classified in 28 conserved families (79 unique predicted mature microRNA sequences).

For the most part, we confirm existing patterns of miRNA family conservation with respect to completely sequenced plant genomes for which extensive analyses of miRNAs have been performed (Arabidopsis thaliana, Populus trichocarpa, Oryza sativa and Physcomitrella patens). Of the 28 families for which we identified putative precursor sequences in Vitis vinifera (Table 1), 9 are represented in all four of these species and a further 10 have been characterized in all three magnoliophytes. One family (miR403) is present in both of the previously sequenced Dicots, while two previously Arabidopsis-specific families (miR828 and miR845) were predicted in grapevine (suggesting their loss or - as yet - undetected presence in poplar), while miR479 and miR482 (annotated only in poplar and grapevine) are likely to have evolved in a common ancestor of these organisms after its divergence from the Arabidopsis lineage [26]. miR477 precursors have been characterized only in poplar, grapevine and $P$. patens, while a series of miR535 precursors represent the first members of this family to be identified in core eudicots, having been identified only in P. patens, rice and more recently in the California poppy (Eschscholzia californica) [28]. The only families tested for which members have been identified in poplar and at least one other of the aforementioned genomes but for which microHarvester failed to identify candidate precursors in the grapevine genome were miR472, miR530 and miR827.

With respect to the reference annotation of protein coding genes in the Vitis vinifera genome, 127 putative pre-miRNAs were intergenic in location (17 overlapped with annotated genes but on the non-coding strand). Four precursor predictions fell within or overlapped annotated coding or UTR exons although homology searches and transcriptomics data generated subsequently to the initial annotations call into question the validity of all but two of these exon annotations. miRNA $156 \mathrm{~h}$ is probably an incorrect prediction derived from a coincidentally plausible hairpin structure formed by the opposite strand to the presumed target (a Squamosapromoter Binding Protein (SBP) box gene). A similar situation is observed for miR171g which falls on the opposite strand to to a GRAS domain transcription factor gene. Nine precursor predictions were apparently intronic in location. Manual checks of the automated annotations suggested that all of the introns putatively containing pre-miRNAs were likely to be erroneous predictions, being atypically long (over $13 \mathrm{~kb}$ ) and interrupting putative retroelement derived genes or obvious fusion gene predictions (not shown).

\section{Deep sequencing of small RNAs from grapevine leaf tissue}

We generated 13,078,222 reads with Illumina sequencing of small RNA isolated from Vitis vinifera L. clone PN40024 leaves. 2,585,821 individual small RNA reads of $18-27$ bases (19.8\% of the total reads generated) yielded at least one perfect match to the draft genome after removal of adapter sequences and allowing for post transcriptional oligoadenylation of reads. After exclusion of reads mapping to annotated structural RNAs, Over 7\% of the total mapped sequences were of length 21 bases and accounted for $7.8 \%$ of the genomic loci represented by the mapped data (mean redundancy of 4.38 reads/locus). $15 \%$ of loci represented were of length 24 (10.7\% of tags sequenced) with a mean redundancy of 3.08 reads/locus, suggesting, in accord with other studies $[29,30]$, that miRNAs in our sample tend to be expressed at higher levels or processed more specifically than the more heterogeneous 24 base small RNAs.

Mapping of the short tags onto the genome sequence revealed that of the 28 families predicted by our comparative analysis, 23 showed at least one sequence tag 
either in exact or very close correspondence to the position of one of the predicted mature sequences (the exceptions being miR395, miR396, miR477, miR828 and miR845). In some cases, the most commonly observed sequences were identical to the predicted mature sequences while for other families, the predominant mature miRNA sequenced exhibited small variations (shifts or differences of length of one or two bases) with respect to the predicted mature sequences. This finding was not unexpected given the variation in mature miRNA lengths within families observed in other plant species and the nature of the comparative method used to generate the initial predictions. For predicted precursors for which matching small RNA reads were recovered, the vast majority of reads conform to the sequences indicated in Table 1 [see also Additional File 1: Supplemental figure S2], consistent with the primary requirement for the annotation of plant miRNA sequences [31].

We recovered a number of reads that include an additional 3' base that does not correspond to any genomic locus, this tendency has been observed in other species (e.g. [29]). Furthermore, a low but notable proportion of mapped small RNA sequences show mismatches to the genomic sequence while preferentially mapping only to putative miRNA precursor loci. This is probably due to errors in sequencing or during reverse transcription or amplification and particularly to the higher error rate of the Illumina sequencing strategy in GC rich sequences [32]. During the course of these analyses it became clear that two precursors (miR172a and 172b) were likely to derive from the opposite strand from that initially predicted. Where appropriate, corrected mature sequences have been deposited in miRBase.

Due to identity or similarity among mature miRNAs belonging to the same family, deep sequencing of small RNAs does not allow consistent unambiguous assignment of mature miRNAs to their genomic loci of origin. Nevertheless our analysis provided indications of the presence and relative abundance of 36 distinct mature miRNAs from conserved families in leaves - corresponding to up to 65 distinct precursor loci (see Table 1 for summary and Additional File 1: Supplemental figure S2 for detailed maps of all small RNA reads mapping to predicted precursors).

\section{Oligonucleotide arrays}

A $12 \mathrm{~K}$ CombiMatrix custom array was developed to validate our in-silico miRNA predictions and to profile miRNAs expression in different tissues.

Slides were hybridized with low molecular weight RNA (LMW-RNA), extracted from six grapevine ( $V$. vinifera L. cv Corvina) tissues: ripening berries (three stages analyzed), roots, leaves and young inflorescences.
Each hybridization and LMW-RNA extraction was performed twice.

In addition to the mature miRNA sequences, the probe set included probes shifted 5 or 10 bases $3^{\prime}$ or $5^{\prime}$ with respect to the central base of the corresponding mature miRNAs as well as probes derived from regions of the stem not predicted to overlap with the mature miRNA sequence and controls containing maximally destabilizing substitutions with respect to probe sequences [see Additional File 2: Supplemental figure S5]. Except for probes shifted 5 nucleotides towards the 5 ' end of the miRNA precursor, for more than $90 \%$ of the probes a signal drop-off greater than $90 \%$ was observed - indicating no significant hybridization for these probes occurred. On the contrary, for probes shifted 5 nucleotide towards the 5 ' end of the miRNA precursor the lack of signal drop-off might be due to the fact that probes were synthesized with their 3' termini towards the slide, and that no "spacer" oligonucleotide was used (according to CombiMatrix protocols). As a consequence, steric effects might reduce the specificity determined by the 3'-most five bases of the probes.

Other than for 26 out of 140 pre-miRNAs (Table 1), no detectable signals were recorded for the probes designed on the precursor loop regions - likely due to size fractionation of RNA samples and the relatively short half-life of pre-miRNAs. We conclude that our miRNA expression data are principally derived from mature miRNAs molecules, without appreciable premiRNA contamination.

Finally, it should be noted that recent studies have demonstrated appreciable levels of cross-hybridization between closely related miRNAs and probes differing by only one or two bases [33]. It is therefore difficult to exclude the possibility that cross-hybridization within miRNA families causes a distortion of quantitative estimates of expression levels of some individual mature miRNA sequences.

\section{Microarray analysis of miRNA expression}

Of the mature miRNA sequences considered, 56 (corresponding to 23 different families), showed significant expression in at least one tissue tested (Table 1 and Additional File 1: Supplemental Figure S1), and another 6 showed a borderline signal. Specifically, 41 different miRNAs showed significant signal in roots, 47 in leaves, 49 in young inflorescences, 53 in green berries, 42 in berries at veraison (the point where growth ends and maturation begins) and 40 in mature berries.

To evaluate the statistical significance of the differential expression of mature miRNAs in the six tissue considered, we set up two distinct comparisons: one among the three developmental stages of the ripening berries 


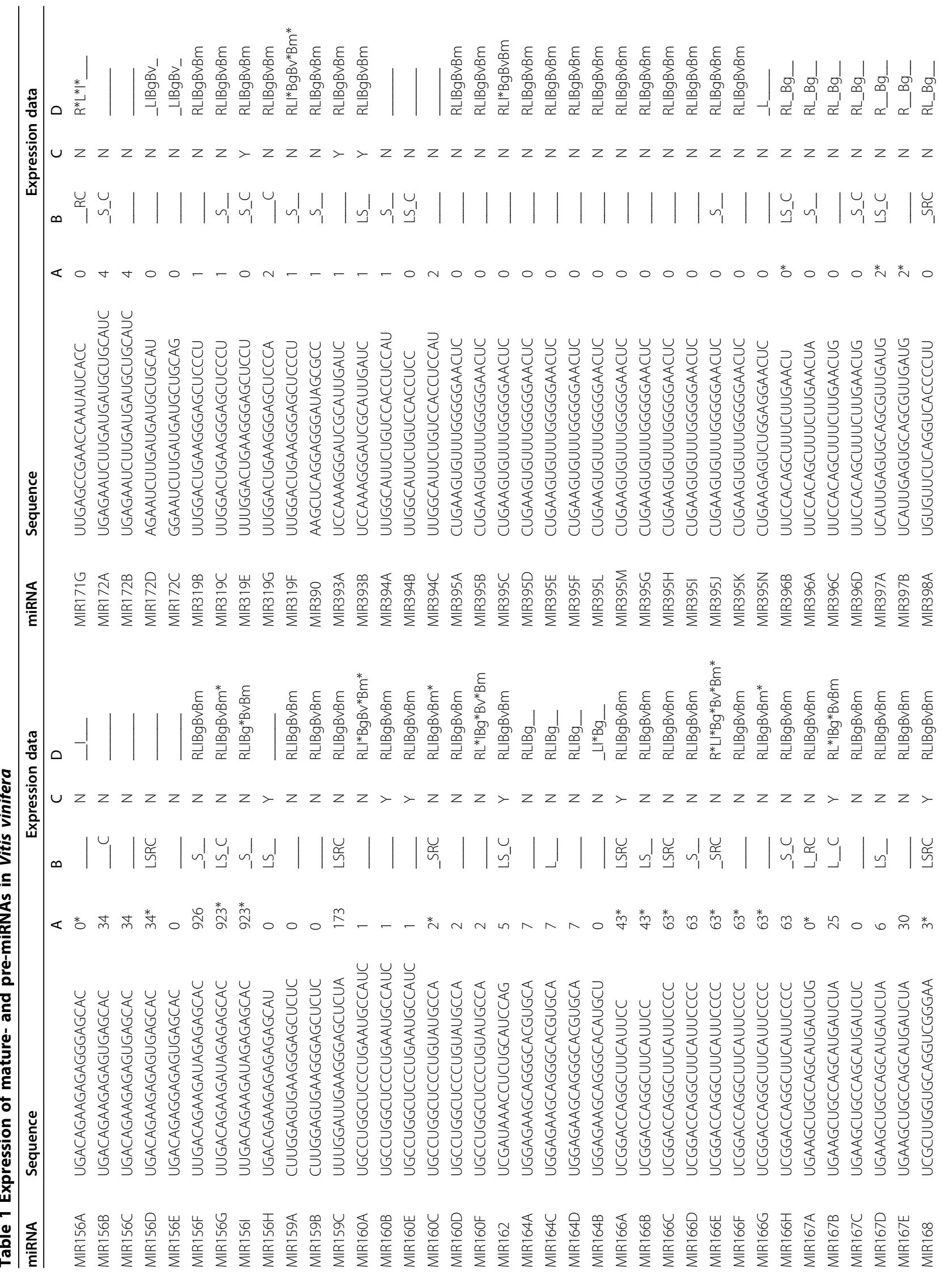




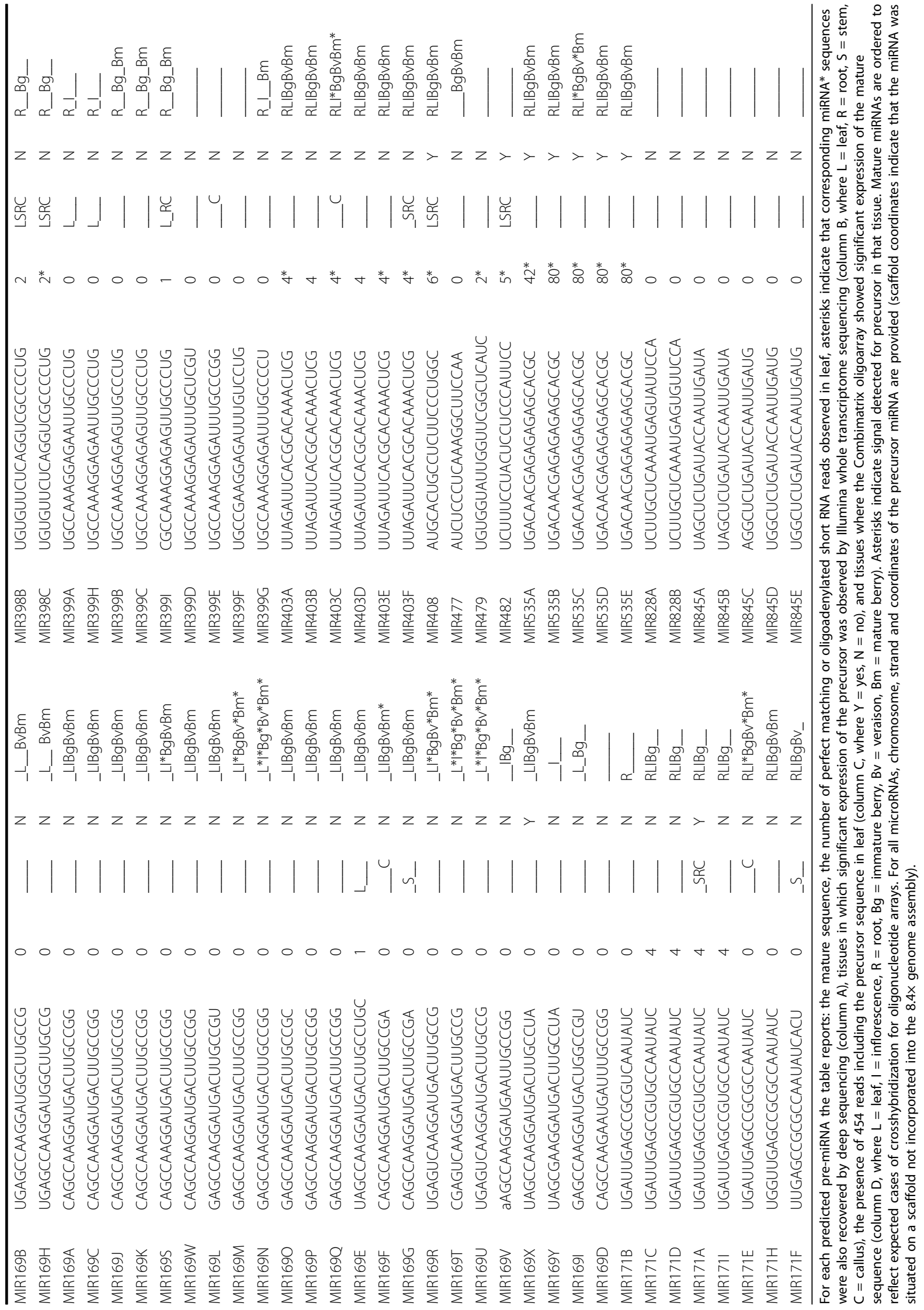




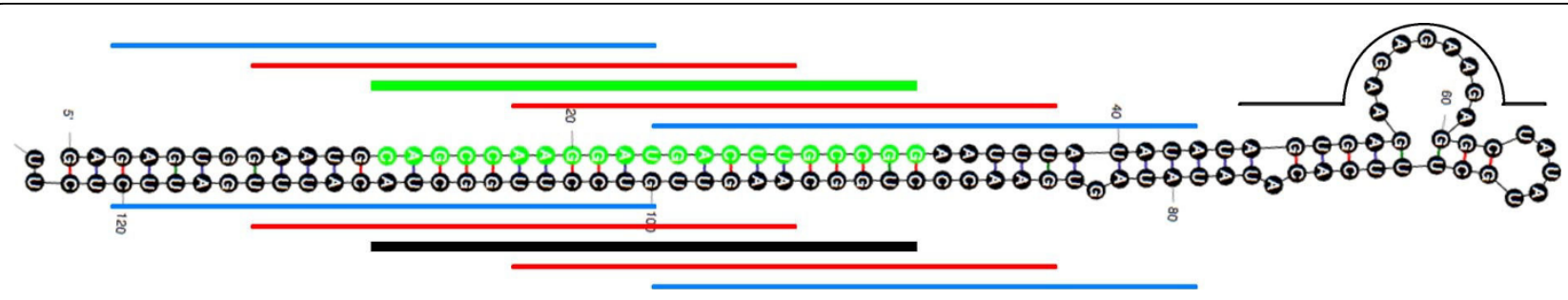

Figure 1 Oligonucleotide design strategy for Combimatrix custom oligonucleotide array. Probes were designed complementary to the predicted mature miRNA (green line) and miRNA* (thick black line) sequences. Additional probes were designed to the loop region (thin black line) as well as probes shifted 5 nucleotides (red lines) and 10 nucleotides (blue lines) with respect to the miRNA and miRNA* sequences.

and the other one among leaves, roots and inflorescences. ANOVA analyses were performed with a Pvalue threshold of 0.05 and subsequently a Scheffè test was used to assess which of the three tissues showed significant differences. Thirteen different mature miRNAs showed a statistically significant change in signal between the ripening stages of the berry (Figure 2A-C), and 27 miRNAs showed significant changes in their expression when comparing three different tissues (leaves, roots and inflorescences)(Figure 2D-H). miR395a and miR171h show a distinctive pattern of expression - being highly expressed at veraison with respect to the other two stages ( 4.4 and 2.3 fold changes of expression level respectively) (Figure 2A). Seven miRNAs (miR156f, miR169a, miR169f, miR169r, miR169x, miR319b and miR535a) are more expressed in mature berries than in green berries (Figure 2B). Four miRNAs (miR171c, miR172c, miR396c, miR403a) are, on the contrary, more expressed in green berries, their expression decreasing during ripening (Figure $2 \mathrm{C}$ ).

Clear patterns also emerge from analyses of differential expression between roots, leaves and young inflorescences. Thirteen miRNAs are significantly differentially expressed in roots, showing a similar expression in the other tissues. In particular miR397a, miR398b and miR408 all show at least 100 fold higher expression in root than either leaf or early inflorescences, while miR159a, miR160a, miR399a, miR399b, miR403a and miR535 show more modest, but still significant, changes in the same comparisons (Figure 2D). On the contrary miR164a, miR164b, miR171c and miR172c show a significantly lower level of expression in roots (Figure 2F).

Five miRNAs (miR169v, miR169y, miR171f, miR171h and miR319b) yield significantly higher signals in young inflorescences than both leaves and roots (between 2 and 7.2 fold higher levels in this tissue)(Figure 2E). Only one miRNA, miR160c, shows a leaf-specific expression profile ( 2.5 fold lower level in leaves with respect to other tissues) (Figure 2G). Finally, six miRNAs (miR169a, miR169e, miR169f, miR169x, miR171e and miR395a) exhibit significant differences in expression levels in all comparisons between leaf, root and inflorescences (Figure 2H). Five of these miRNAs (169a, $169 \mathrm{e}, 169 \mathrm{f}, 169 \mathrm{x}$ and 171e) show the highest expression in young inflorescences and the lowest in roots.

Following the widespread assumption that many miRNA/target interactions are conserved between related species [2,3], our data regarding differential expression of mature miRNA sequences raise some intriguing possibilities particularly with respect to the potential importance of miRNA in the regulation of fruit maturation.

Li et al. [34] recently showed that the transcription factor NFYA 5 is targeted by miR169 and that overexpression of miR169 leads to excessive water loss through leaves and hypersensitivity to drought stress in A. thaliana. In this light, the preponderance of miR169 family members in the group of miRNAs upregulated in mature berries is striking and might reflect a mechanism to protect maturing fruit from dehydration. We also note that miR535 family, identified so far only in $O$. sativa and $P$. patens [35] is upregulated during berry maturation. This is a first indication of a possible function of miR535 for which no information was previously available. miR396c shows 6 fold decrease in expression during ripening. The mir396 family targets seven Growth Regulating Factor (GRF) genes in Arabidopsis [14]. GRF genes encode putative transcription factors associated with cell expansion in leaf and other tissues in A. thaliana and O. sativa [36,37]. A potential role for miR396 in the regulation of cell expansion during fruit maturation is an intriguing hypothesis. In addition, recent data also link miR396 to responses to abiotic stresses including drought [38], again suggesting the importance of water homeostasis during berry ripening. miR172, downregulated during berry maturation, targets Apetala 2 (AP2) -like transcription factors, regulators of flowering time, organ identity and of vegetative phase change [39]. In grapevine, genes related to AP2 are upregulated at veraison, being involved in berry maturation [40] and putatively connected with abiotic and biotic stress resistance. This evidence fits well with our findings. The sharp up-regulation of miR395 at veraison suggests a further role for miRNAs in an agronomically 




Figure 2 Differential expression of mature miRNAs by tissue. miRNAs showing significant changes in expression by tissue are reported. Panels A-C: miRNAs differentially expressed in one stage of berry ripening A: at veraison, B: in green berries, C: in mature berries. Panel D: miRNAs more highly expressed in roots, Panel E: miRNAs more highly expressed in inflorescences, Panel F: miRNAs less expressed in roots, Panel G: miRNAs less expressed in leaves, Panel H: miRNAs showing significant differences in all tissues tested. Error bars indicate confidence intervals. For all panels, the $Y$ axis shows Log2 of the normalized median of spot intensities.

important aspect of grape maturation. miR395 is known to contribute to the regulation of sulfur metabolism, targeting both sulfate transporters and ATP sulphurylase genes. A direct connection between ATP sulfurylases and berry maturation has not been demonstrated, but it is known that a Glutathione S-transferase is strongly connected with berry ripening and in particular with coloration during berry development [40].

miR397a, miR398b and miR408 which are extremely highly expressed in root tissues target various copper proteins: plantacyanin, laccases and a superoxide dismutase, all putatively involved in stress responses and lignification $[14,16,41,42]$. These miRNAs have also been shown to be coexpressed in Arabidopsis under conditions of copper deprivation [43]. Moreover some laccase genes in Arabidopsis are root specific (for example
AtLAC15) or mostly expressed in roots [44] and are involved in root elongation and lignification [45]. Given that grapevine roots are much more lignified than those of Arabidopsis, it is plausible that regulation of laccase expression is vital in the grapevine. It is interesting to note that the laccase family is, along with other polyphenol oxidase gene families, massively expanded in grapevine with respect to Arabidopsis (>60 genes in $V$. vinifera, 17 in Arabidopsis).

\section{Whole transcriptome sequencing and differential expression of precursors}

The majority of plant miRNA genes are transcribed by RNA polymerase II and result in the production of polyadenylated primary transcripts [8]. A strict correlation between expression levels of individual precursors and 
levels of mature miRNAs should not be expected. Mature miRNAs are likely to be, in general, more stable than their corresponding primary transcripts and may derive from more than one genomic locus. Furthermore, recent data in plants [46] and animals [47] suggest that a variety of mechanisms, including alternative splicing and the specific binding of protein factors, can regulate the efficiency with which pri- or pre-miRNAs are processed. High levels of primary transcript can thus be associated with low levels of mature miRNAs and viceversa. These considerations notwithstanding, it is reasonable to presume that sequences derived from highly expressed pri-miRNA transcripts should be represented in whole transcriptome "deep sequencing" experiments.

To investigate this hypothesis, we have analyzed whole polyA $^{+}$transcriptome data generated with the Illumina Solexa technology [48] and Roche 454 next generation sequencing platforms.

A total of 135,047,735 Illumina sequences (33-35 bases in length) derived from polyA ${ }^{+}$RNA isolated from 4 tissues (in vitro cultivated juvenile leaf $(29,829,113$ sequences), in vitro cultivated juvenile stem $(30,785,175$ sequences), in vitro cultivated juvenile root $(29,254,635$ sequences) and embryonic callus (45,178,812 sequences) were mapped to the grapevine genome and coordinates compared to those for predicted pre-miRNAs.

The statistical significance of the number of reads mapping within a predicted pre-miRNA was evaluated (see Materials and Methods) and 52 predicted precursors show significant expression in at least one tissue (25 in leaf, 38 in stem, 17 in root, 33 in callus)(Table 1). Many predicted precursors show a wide expression (miR156d, miR159c, miR166a and c, miR168, miR171a, miR398a, miR398b and c, miR408, miR482). In some families, when expressed, precursors show overlapping patterns. For example, miR319c, miR319e and miR319f are all expressed in stem, while miR319c and miR319g are expressed in callus, no expression of miR319 was detected in leaf or root. A similar situation is observed for the miR396 family. In other cases, different precursors seem to be predominantly expressed in different tissues. For example miR171e transcripts are detected only in callus, miR171f is only transcribed in stem while miR171g is observed in callus and root - a similar situation can be observed for several families including miR166, miR167 and miR169). These data suggest that tissue specific expression of different precursors within single families is widespread in the grapevine.

454 sequencing generated 613,098 and 581,655 reads respectively from leaf and berry polyA ${ }^{+}$RNA. The expression of 15 unique predicted precursor sequences received ulterior support from these data (Table 1). With the exception of miR160b and the miR535 family the expression of all precursors detected by 454 sequencing in leaf was also strongly supported by Illumina data. Interestingly, given the lack of detectable expression of the mature sequence in leaf or berry, miR482 precursors were detected at high levels both by Illumina and 454 sequencing, suggesting post-transcriptional regulation of processing of this transcript.

\section{Estimation of primary microRNA transcripts and splice sites}

For a number of predicted microRNAs the density of coverage of the corresponding genomic loci was sufficient to attempt to estimate primary transcript coordinates as well as patterns of splicing and alternative splicing.

We constructed Position Specific Scoring Matrices (PSSMs) of experimentally validated grapevine canonical splice donor and acceptor contexts (French-Italian Consortium for Characterization of the Grapevine Genome, unpublished data) and used these matrices to evaluate all possible canonical splice donors and acceptors from $3 \mathrm{~kb}$ upstream to $3 \mathrm{~kb}$ downstream of predicted microRNA precursors showing extensive coverage by Illumina RNA-Seq reads. The positions and flanking exonic sequences of all possible splice donor/acceptor pairs were used to combinatorially generate possible splice junctions. RNA-Seq reads which did not map perfectly to the genome sequence were compared to these computationally generated splice junctions and pairs providing perfect matches (with at least 8 bases on either side of the splice junction) were recorded along with the tissue distribution of reads supporting each splice event. Additionally, for each supported splice event, we recorded the ratio of base coverage by RNA-Seq of the flanking 40 putatively exonic bases and the coverage of flanking 40 putatively intronic bases (not including reads previously identified as covering the putative splice junction). Three fold greater coverage of exonic regions was considered as additional support for the presence of a functional splice junction. Introns inferred from mapping of 454 transcriptome reads were also recorded.

Visual examination of RNA-Seq coverage of regions upstream and downstream of miRNA precursor loci was used to provide initial estimates of transcript start and end positions. This step was complicated by the known propensity of RNA-Seq to provide uneven coverage of transcript termini - presumably due to the dynamics the nebulization step in sample preparation and to issues associated with differential recovery of fragments during sample preparation. Accordingly, we subjected the $6 \mathrm{~kb}$ interval centered on predicted precursors to promoter prediction analysis by TSSP-TCM [49] in order to attempt to provide support for manually identified transcription start sites. Estimated transcript coordinates, putative intron coordinates quality scores for each 


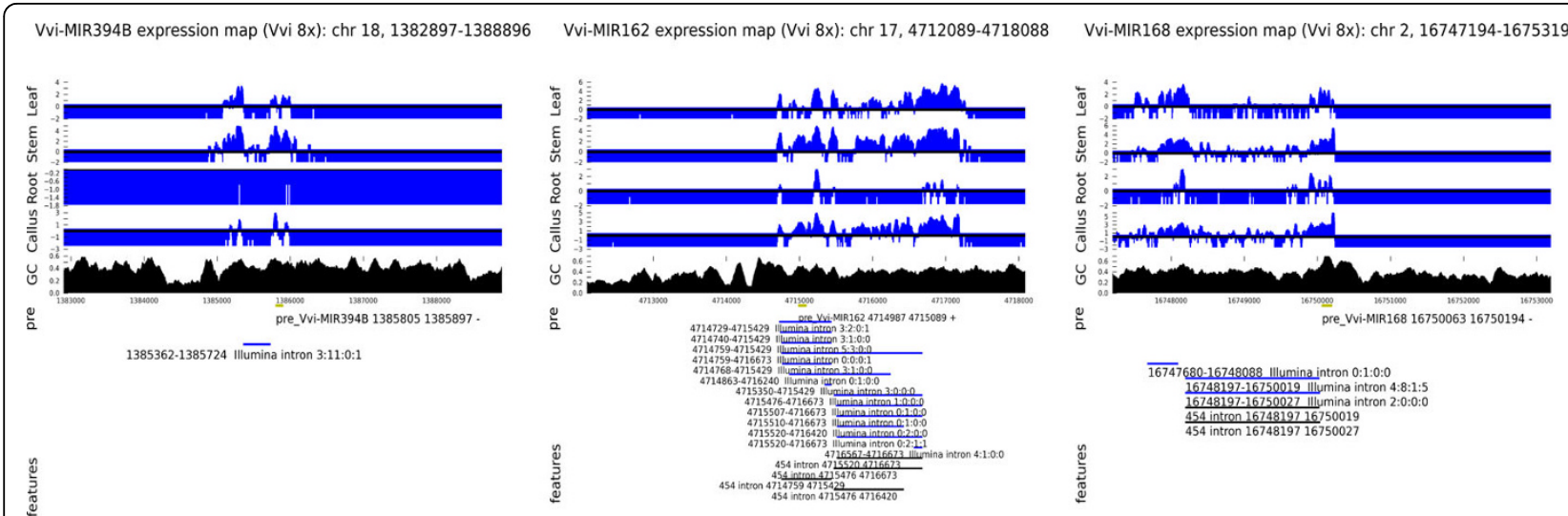

Figure 3 Transcription and splicing of pri-miRNAs in Vitis vinifera. A summary of transcription of genomic loci containing predicted premiRNAs is provided. Illumina whole transcriptome reads per base are reported for four tissues as log(number of reads/expected number of reads under random distribution of reads). Local GC content, position and strand of predicted pre-miRNA as also shown along with coordinates of: canonical introns inferred from non-contiguous mapping of Illumina reads (blue bars), 454 reads (black bars) and assembled 454 sequence contigs (green bars). Predicted genes where present are represented by red bars. Panel A refers to miR394B, panel B to miR162 and panel C to miR168.

donor/acceptor, frequencies of splice junction-covering reads, and TSS proposed by TSSP-TCM are reported in Additional File 1: Supplemental Table S4.

Figure 3 shows the transcriptional landscape for the genomic region from $3 \mathrm{Kbp}$ upstream to $3 \mathrm{Kbp}$ downstream of three exemplar predicted miRNA precursors, including introns inferred from 454 and Illumina sequence data, the concordance of splicing events identified by 454 and Illumina reads is notable and consistent with the reliability of the Illumina data to infer splicing events. Detailed genomic alignments of all reads supporting splices indicated in Figure 3 are available in Additional File 1: Supplemental Figure S4. We note that relative numbers of tags representing different regions of putative primary miRNA transcripts vary but tend to be consistent between different tissues. The GC content of 100 base windows centered on each genomic position are also shown and illustrate, within the proposed primary transcripts, an apparent correspondence between depth of coverage and GC content [32].

Figure 3A shows the transcriptional context of miR394b and the presence of a canonical intron supported by 14 Illumina reads ( 7 distinct sequences). This intron was also easily detectable through RACE experiments (see Additional File 1: Supplemental Table S3). We note that the position of the intron corresponds well to a region of low, or undetectable levels of Illumina transcriptome coverage, and that tissue specific differences in Illumina reads mapping to this region are quite apparent. These data suggest that our approach is capable of identifying introns in pri-miRNA transcripts and differences in steady state levels of pri-miRNA transcripts between tissues. Additionally, 3' RACE experiments indicated a transcript 3' end within $20 \mathrm{bp}$ of the position predicted from RNA-Seq read coverage (see Additional File 1: Supplemental Table S3).

The miR162 precursor (Figure 3B) is of particular interest in that it covers a region including several potential canonical introns that are supported by multiple Illumina and 454 reads. All tissues indicate that the transcriptional start site falls between positions $4,714,680$ - 4,714,687 on chromosome 17. The postulated canonical introns imply alternative splicing of the nascent primary transcript from this locus, as does the coverage of the region by 454 contigs (whose map positions are also consistent with the Illumina data with respect to the overall coordinates of the nascent primary transcript). Several of these introns, and the alternative splicing of this transcript were also supported by preliminary RACE experiments (see Additional File 1: Supplemental Table S3). Indeed, while the boundaries of proposed introns correspond to "shoulders" of falling transcript coverage, significant levels of reads mapping within the introns are observed. Interestingly, Hirsch et al. [46] recently demonstrated that the primary miR162a transcript of Arabidopsis is subjected to complex pattern of alternative splicing, similar to that proposed for the grapevine miR162 transcript. In Arabidopsis, only unspliced isoforms are capable of yielding mature miRNAs. Our findings therefore suggest conservation of alternative splicing as a key regulatory mechanism in miR162 expression and indicate that Illumina and 454 transcript data can also be used to identify alternatively spliced plant pri-miRNAs.

Figure 3C shows evidence for expression of the miR168 locus. Analogously to miR162, our data suggest 
alternative splicing of the pri-mRNA, while the distribution of 454 contigs is highly consistent with the Illumina data. Vaucheret et al. [50] showed that AGO1, the target of miR168 is involved in the regulation of miR168 stability. Our data may hint at yet another mechanism of regulation of this intriguing miRNA.

Of 25 precursor loci chosen on the basis of extensive RNA-Seq coverage (see Additional File 1: Supplemental Table S3), 18 showed evidence of transcript splicing and 8 of alternative splicing, suggesting that post-transcriptional modification of miRNA transcripts is likely to be widespread. It is possible that some splicing events frequently identified by deep sequencing approaches could be associated with regulation of downstream processing of transcripts as has been shown for the miR162 transcript of Arabidopsis [46]. For miR162 and miR168, this hypothesis might be consistent with the low levels of mature microRNA observed by deep-sequencing, in contrast to the apparently high spliced transcript levels. For several pre/pri-miRNA loci (notably miR162 and miR168) we infer several closely related canonical introns (shared splice donors with splice acceptor sites shifted by a few tens of bases or vice-versa). We speculate that this phenomenon might be due, in part, to the incapacity of the Nonsense Mediated Decay pathway (which is dependent on ribosomal scanning of mRNAs [51] to monitor "erroneous" splicing of non-coding transcripts.

The estimation of primary transcript coordinates, and in particular transcription start sites is a critical step towards the elucidation of specific mechanisms regulating the expression of miRNAs at the transcriptional level. Our Illumina transcript reads are non-directional it is not possible to establish from which strand of the genome a transcript is derived. However, we show elsewhere that both concomitant transcription of both genomic strands at single loci and transcription of intergenic regions are rare in grapevine (French-Italian Consortium for Characterization of the Grapevine Genome, unpublished data). Thus, evidence of transcription of intergenic pre-miRNAs can reasonably be considered as validation of transcription of the precursor.

The finding that relative depth of coverage of different regions of primary transcripts is consistent between tissues suggest the presence of systematic biases in either the procedure used to fragment the cDNA, in amplification of fragments for sequencing, or in sequencing efficiency. Dohm et al. [32] observed a strong relationship between local GC content and depth of coverage with Illumina genome resequencing. Indeed, we observe a relationship between local GC content and depth of coverage - even within regions that show contiguous coverage and are unlikely to represent introns (correlation between log coverage for positions represented by at least one sequence and GC content of 100 base window centered on that position for all bases within $3 \mathrm{~kb}$ of a predicted precursor is $>0.25$ for all tissues, $\mathrm{p}=0$ ). However, grapevine introns between both coding and non-coding exons show a low GC content (34.7 and $32.3 \%$ respectively) with respect to coding and non-coding exons (44 and $37.3 \%$ respectively) [26]. Thus, it may be difficult to differentiate between introns and regions where low coverage is a result of low GC content in exonic regions on the basis of Illumina transcriptome data - particularly where levels of template are likely to be low and a-priori gene models are not available. However, the discovery that putative splice junctions for primiRNAs can be identified by discontiguous mapping of illumina reads may help to ameliorate this problem for plant pri-miRNAs. The fact that we recovered evidence of alternative splicing of miR162, is consistent with data from $A$. thaliana [46] and validates our basic approach. Indeed, other putative pri-miRNAs, including miR394b show evidence of splicing from both transcript coverage and discontiguous mapping of whole transcriptome reads.

\section{Conclusions}

We have used a combination of high throughput approaches to show highly tissue specific expression of mature miRNAs in the grapevine Vitis vinifera including the first evidence of differential expression of miRNAs during fruit maturation in this species. We have shown that, for plants at least, whole transcriptome sequence data can be applied to the detection of differential transcription of putative precursor miRNA loci and to the detection and definition of pri-miRNAs as well as to the tentative definition of patterns of splicing in such precursors. It is probable that similar analyses performed in lines carrying mutations in genes involved in miRNA processing (in particular DCL1- plants) will allow more extensive and accurate definition of miRNA transcripts on a large scale, eventually facilitating detailed analyses of promoter sequences and a deeper understanding of mechanisms of transcriptional regulation of miRNA genes. Our analyses also suggest that splicing (and alternative splicing) of pri-miRNAs may be widespread and might constitute a general mechanism for the regulation of miRNAs.

\section{Methods}

\section{Plant materials}

Grapevine (Vitis vinifera L.) clone PN40024 plants and callus tissue were cultivated in vitro under standard conditions. For oligonucleotide array analyses, fresh tissues, with exception of roots (where in vitro cultivated plants were used), were collected from field-grown $V$. vinifera L. cv Corvina. Grape berries were harvested 5, 9 and 15 
weeks after flowering, while leaves were collected from pre-flowering plants, inflorescence samples were collected 3 weeks before flowering.

\section{RNA extraction and deep sequencing}

For Illumina deep sequencing, total RNA from PN40024 was extracted with the Spectrum Plant Total RNA Kit (SIGMA) as directed by the manufacturer. RNA was DNase treated with RQ1 RNAse-Free DNAse (Promega) and RNA integrity was checked using an Agilent Technologies 2100 Bioanalyzer.

Total RNA samples from leaf, root, stem and callus were processed using proprietary kits at Illumina, Inc. in Hayward (CA, USA). Briefly, PolyA ${ }^{+}$RNA was isolated from total RNA fragmented using Ambion RNA fragmentation buffer. cDNA synthesis was performed with Invitrogen random hexamer primers and cDNA was purified using QIAquick PCR spin column (Qiagen). Ends were blunted and 3' A overhangs introduced using T4 DNA polymerase and E. coli DNA polymerase I Klenow fragment. cDNAs were ligated to adapters with a single ' $T$ ' base overhang. After selection of 150-200 bp fragments from $2 \%$ low-range agarose gel, samples were amplified by 18 PCR cycles to enrich cDNAs with correctly ligated adapters and to amplify the amount of DNA in the library. Samples were loaded on Cluster Station to create flow cells of CSMA (Clonal Single Molecular Array) and sequenced on the Illumina platform. RNA-Seq data are available from http://www. genoscope.cns.fr/externe/gmorse/raw_data/.

Small RNAs (20-30 nt) were isolated from leaf total RNA by a denaturing PAGE gel. Samples were prepared for sequencing using proprietary kits at Illumina, Inc (CA, USA). Briefly, 5' and 3'-adapters were ligated to small RNAs. After reverse transcription, a low number of PCR cycles were used to create a sufficient amount of cDNA constructs. cDNA sample was then loaded on the Cluster Station and sequenced at ultra-high throughput on the Illumina platform. Small RNA sequencing data are available from the Short Reads Archive (SRA) under accession number SRS005164.

For 454 transcriptome analysis, polyA ${ }^{+}$RNA was isolated from $V$. vinifera L. cv Corvina leaf and berry tissues by according to Rezaian and Krake [52]. After reverse transcription using an oligo(dT)-adapter primer for first strand synthesis, cDNAs were amplified with 18 (leaf) and 17 (berry) cycles of LA-PCR [53]. Normalization was carried out by one cycle of denaturation and reassociation of the cDNA. Reassociated ds-cDNA was separated from the remaining ss-cDNA (normalized cDNA) by passing the mixture over a hydroxylapatite column. After hydroxylapatite chromatography, the sscDNA was amplified with 9 LA-PCR cycles using phosphorylated primers. cDNAs were finally purified using the NucleoSpin ExtractII kit from Macherey \& Nagel and subjected to sequencing on the ROCHE 454 GS FLX platform according to manufacturer's instructions.

For oligonucleotide array analyses, total RNA was extracted from $V$. vinifera $\mathrm{L}$. cv Corvina tissues and size fractioned, following the procedure [54] with minor modifications. Before extracting RNA from berries, seeds were separated from the rest of the fruit. Low molecular weight (LMW) RNA was checked for quality and quantity using the NanoDrop Spectrometer (ND 1000, Celbio SpA) and the Agilent 2100 Bioanalyazer.

\section{Oligonucleotide arrays}

Predicted grapevine miRNA precursor sequences have been published elsewhere (Jaillon et al., 2007). A CombiMatrix $12 \mathrm{~K}$ CustomArray was synthesized with 1947 miRNA-specific probes synthesized to test grapevine miRNA expression profiles.

For each grapevine miRNA precursor, we designed a set of $20-22 \mathrm{nt}$ probes specific for the mature miRNA, the miRNA* and their complementary sequences, as well as a probe specific for the non mature microRNA stem/loop region and probes designed on the miRNA and miRNA* sequences but shifted of five or ten nt, forward and backward in order to test probe specificity. snRNA U6 and four grapevine tRNA probes were used as positive controls. Fourteen distinct negative and mRNA degradation control probes were included. Additionally, for each specific probe, a mismatch control with 2 maximally destabilizing substitutions was included. Each probe was present on the final array in three replicates. All probe sequences are available in Additional File 2: Supplemental Table S6.

Slides were hybridized with $3 \mathrm{~g}$ of LMW RNA labeled with Cy5 (Mirus LabelIT miRNA labeling Kit (Mirus Bio Corp.)). Hybridization and washing were performed as indicated by CombiMatrix. Slides were scanned with a Perkin Elmer Scanarray 4000 XL raw data was extracted with Scanarray Express 4.0 and Microarray Imager (CombiMatrix) software. After each hybridization, slides were stripped according to manufacturer's instructions and re-used 5 to 6 times.

Two hybridizations were performed with independently extracted LMW RNAs, for each sample. Background level was defined as the average signal of the negative and degradation controls plus two times their standard deviation. The ratio between intensities of the perfect match probe and its mismatch probe (referred to as $\mathrm{PM} / \mathrm{MM}$ ) was also used to estimate the reliability of each signal. Probes with a median signal higher than background and with PM/MM value higher than 1.2 were called as present. The normalization between arrays was performed using the quantile normalization 
method [55] using the BLIST software, provided by Combimatrix.

Normalized signals were Log2 transformed and probes with a low PM/MM ratio $(<1.2)$ were discarded. Differentially expressed genes in various tissues were identified with a one-way ANOVA test ( $p$-value $<0.05)$. Significant results were further investigated with Scheffè test, a post hoc test to define which tissues showed significant differences.

The use of short RNA probes has not proven to be effective to distinguish between miRNAs that have few differences, in particular at the first or last nucleotide [33], therefore microarray data from closely related miRNAs have been treated as replicated data. Thus different miRNA precursors that give rise to almost identical mature products have been clustered as single entities.

Complete oligonucleotide array experimental design and data are available from the Gene Expression Omnibus [GEO: GSE13801].

\section{RACE experiments}

Total RNA was extracted from $V$. vinifera L. cv Corvina leaves, using a rapid CTAB method, as described [56], with minor modifications, and DNAse treated (DNase I from Sigma). The FirstChoice RLM-RACE kit (Ambion) was used to perform classic 3' and 5' RACE protocols, following manufacturer's instructions. PCR products, obtained with gene specific primers (miR482-5'-Rout-CGGCATAGGATCTGAGTCCAC, miR482-3'-Finn-AGGAAGAATGGTGGATTCATTA, miR394b-5'-Rout-CCTCTTTTGTGGCTGTGAGATG, miR394b-5'-Rinn-TGAAAGAGGCAAAGAGGAGGAG, miR394b-3'-Fout-CAATCTCTCTCGCTCTTCCACT, miR394b-3'-Finn-ACATCTCACAGCCACAAAAGAG, miR162-5'-Rout-GAATTTGGCGTTGTGATGCTAC, miR162-5'-Rinn-AGAAGAACACAGGGCGGATCT, miR162-3'-Fout-AGACTCTGGTAGCATCACAACG, miR162-3'-Finn-GGTTTATCTTCCGATGGAGAAC), were subsequently cloned in pGEM-T Easy vector (Promega) and sequenced.

\section{Bioinformatics methods}

For small RNA deep sequencing, initial reads of length 33 bases were scanned for the presence of the 5' part of the 3 ' linker sequence 5'-TCGTATGCCGTCTTCTGCTTG3 ' allowing 2 mismatches. Raw sequences whose last 9-14 bases represented the first 9-14 bases of the 3' cloning primer were mapped to the draft genome sequence after removal of adaptor sequences using the software SOAP [57]. Whole transcriptome Illumina reads were mapped to the Vitis vinifera genome using the software SOAP.

Counts of reads mapping to defined genomic loci and all statistical analyses of cluster densities were performed using custom scripts written in PYTHON.
For Illumina transcriptome data, we estimate the probability that at least the observed number of reads should be clustered in the genomic interval defined by the precursor using the Poisson distribution. Thus, we exclude all reads mapping to predicted genes, and search for significant violations (at the $1 \%$ confidence interval) of the null-hypothesis that remaining reads should be distributed randomly among intergenic regions. We consider only reads mapping uniquely to a single genomic locus. Given the expected short half-life of most primary miRNA transcripts, we believe that these criteria constitute an extremely conservative test of precursor expression.

For the genomic regions containing predicted miRNA precursors, we used ad-hoc PYTHON scripts exploiting the MatPlotLib library to plot, for each base the log of the coverage (normalized to the expected coverage under a null model of random distribution). Thus only values above zero reflect higher than expected numbers of transcript-derived matches.

Python scripts were used to generate PSSMs for U2 splice donors and acceptors (3 exonic bases, 14 intronic bases) and aggregate log scores were used to assign scores to all possible canonical splice donors and acceptors. Custom PYTHON scripts were used to combinatorially generate putative splice junctions and SOAP was employed to map RNA-Seq reads that did not provide contiguous perfect matches to the genome sequence to such junctions.

454 and RACE reads were mapped to the Vitis vinifera $\mathrm{L}$. genome sequence using preliminary Blast searches and fine mapping of splice junctions was performed using SPIDEY [58] with default settings for plant sequences. Only reads where over $85 \%$ of the read length was aligned at over $95 \%$ identity were considered.

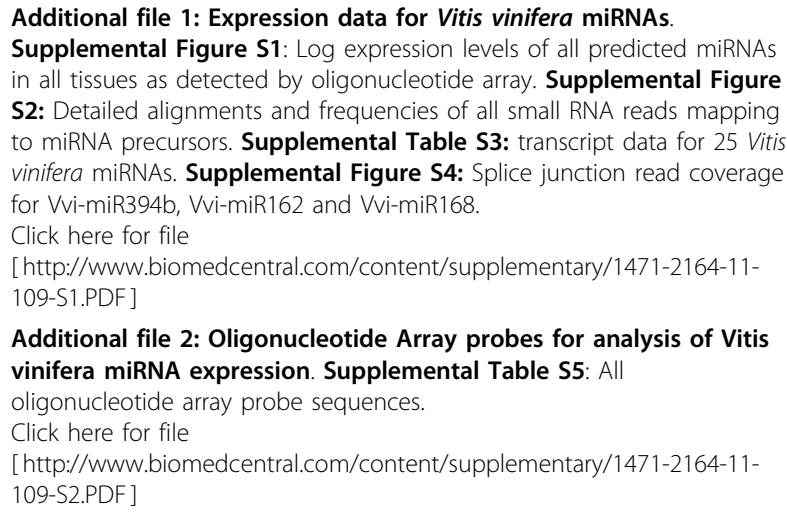

Additional file 2: Oligonucleotide Array probes for analysis of Vitis vinifera miRNA expression. Supplemental Table S5: All

oligonucleotide array probe sequences.

Click here for file

[http://www.biomedcentral.com/content/supplementary/1471-2164-11109-S2.PDF ]

\section{Acknowledgements}

This work was conducted within a scientific cooperation agreement between the French and Italian Ministries of Agriculture and involving: 
Consiglio per la Ricerca e Sperimentazione in Agricoltura (CRA), Regione Friuli Venezia Giulia and I'Institut National de la Recherche Agronomique (INRA). This work was financially supported mainly by the Ministero Politiche Agricole e Forestali, Friuli Innovazione, Federazione BCC, Fondazione CRUP, Fondazione Carigo, Fondazione CRT, Vivai Cooperativi Rauscedo, Eurotech, Livio Felluga, Marco Felluga, Venica e Venica, Le Vigne di Zamò (IGA). Additional financial support was provided by Fondazione Cariverona Project "Centro di Genomica Funzionale Vegetale", Fondazione Monte dei Paschi di Siena and Fondazione Cassa di Risparmio di Lucca.

\section{Author details}

'Dipartimento di Scienze Biomolecolari e Biotecnologie, Università degli Studi di Milano, Milano, Italy. ${ }^{2}$ Scuola Superiore Sant'Anna, Pisa, Italy. ${ }^{3}$ Dipartimento di Biotecnologie, Università degli Studi di Verona, Verona, Italy. ${ }^{4}$ Dipartimento di Scienze Tecnologie e Mercati della Vite e Vino, Università degli Studi di Verona, Verona, Italy. ${ }^{5}$ Dipartimento di Scienze Agrarie ed Ambientali, Università degli Studi di Udine, Udine, Italy. ${ }^{6} \mathrm{CRIBI}$ Biotechnology Centre, Dipartimento di Biologia, Università degli Studi di Padova, Padova, Italy. ${ }^{7}$ Istituto di Genomica Applicata, Udine, Italy. ${ }^{8}$ Istituto Tecnologie Biomediche, Consiglio Nazionale delle Ricerche, Bari, Italy. 'Dipartimento di Biochimica e Biologia Molecolare "E. Quagliariello", Università di Bari, Bari, Italy.

\section{Authors' contributions}

EM Extracted RNA for oligonucleotide arrays, performed oligonucleotide array experiments and analyses of oligonucleotide array data and contributed to drafting the manuscript. VP designed the oligonucleotide array, performed analyses of deep sequencing data and contributed to drafting the manuscript. MEP conceived the project, provided technical support and contributed to drafting of the manuscript, MD and AF performed oligonucleotide array experiments and data extraction, MP provided plant biomass, CC performed analyses of oligonucleotide data, GV provided 454 sequence data, CDF and AP provided software for mapping of splice junctions, MM provided Illumina short sequence data, GP supervised the project, provided technical support and contributed to drafting the manuscript. DSH provided software for data analyses, performed data analyses, supervised the project and contributed to drafting the manuscript. All authors have read and approved the final manuscript.

\section{Received: 12 February 2010}

Accepted: 12 February 2010 Published: 12 February 2010

\section{References}

1. Mica E, Piccolo V, Delledone M, Ferrarini A, Pezzotti M, Casati C, Del Fabbro C, Valle G, Policriti A, Morgante M, Pesole G, Pe ME, Horner DS High throughput approaches reveal splicing of primary microRNA transcripts and tissue specific expression of mature microRNAs in Vitis vinifera. BMC Genomics 2009, 10(1):558.

2. Bartel DP: MicroRNAs: Genomics, Biogenesis, Mechanism, and Function. Cell 2004, 116(2):281-297

3. Jones-Rhoades MW, Bartel DP, Bartel B: MicroRNAs and their regulatory roles in plants. Annual Review of Plant Biology 2006, 57(1):19-53.

4. Mallory AC, Vaucheret $\mathrm{H}$ : Functions of microRNAs and related small RNAs in plants. Nat Genet 2006, 38(Suppl):S31-36.

5. Chen X: A microRNA as a translational repressor of APETALA2 in Arabidopsis flower development. Science (New York, NY) 2004, 303(5666):2022-2025.

6. Dugas DV, Bartel B: MicroRNA regulation of gene expression in plants. Current opinion in plant biology 2004, 7(5):512-520.

7. Allen E, Xie Z, Gustafson AM, Carrington JC: microRNA-directed phasing during trans-acting siRNA biogenesis in plants. Cell 2005, 121(2):207-221.

8. Xie Z, Allen E, Fahlgren N, Calamar A, Givan SA, Carrington JC: Expression of Arabidopsis miRNA genes. Plant Physiol 2005, 138(4):2145-2154.

9. Han MH, Goud S, Song L, Fedoroff N: The Arabidopsis double-stranded RNA-binding protein HYL1 plays a role in microRNA-mediated gene regulation. Proc Natl Acad Sci USA 2004, 101(4):1093-1098.

10. Kurihara $Y$, Watanabe $Y$ : Arabidopsis micro-RNA biogenesis through Dicerlike 1 protein functions. Proc Natl Acad Sci USA 2004, 101(34):12753-12758.

11. Park W, Li J, Song R, Messing J, Chen X: CARPEL FACTORY, a Dicer Homolog, and HEN1, a Novel Protein, Act in microRNA Metabolism in Arabidopsis thaliana. Curr Biol 2002, 12(17):1484-1495.
12. Vaucheret $H$, Vazquez $F$, Crete $P$, Bartel DP: The action of ARGONAUTE1 in the miRNA pathway and its regulation by the miRNA pathway are crucial for plant development. Genes Dev 2004, 18(10):1187-1197.

13. Dezulian T, Remmert M, Palatnik JF, Weigel D, Huson DH: Identification of plant microRNA homologs. Bioinformatics 2006, 22(3):359-360.

14. Jones-Rhoades MW, Bartel DP: Computational Identification of Plant MicroRNAs and Their Targets, Including a Stress-Induced miRNA. Molecular Cell 2004, 14(6):787-799.

15. Llave C, Kasschau KD, Rector MA, Carrington JC: Endogenous and Silencing-Associated Small RNAs in Plants. Plant Cell 2002, 14(7):1605-1619.

16. Lu S, Sun Y-H, Shi R, Clark C, Li L, Chiang VL: Novel and Mechanical StressResponsive MicroRNAs in Populus trichocarpa That Are Absent from Arabidopsis. Plant Cell 2005, 17(8):2186-2203.

17. Reinhart BJ, Weinstein EG, Rhoades MW, Bartel B, Bartel DP: MicroRNAs in plants. Genes Dev 2002, 16(13):1616-1626.

18. Sunkar R, Girke T, Jain PK, Zhu J-K: Cloning and Characterization of MicroRNAs from Rice. Plant Cell 2005, 17(5):1397-1411.

19. Sunkar R, Girke T, Zhu J-K: Identification and characterization of endogenous small interfering RNAs from rice. Nucl Acids Res 2005, 33(14):4443-4454.

20. Sunkar R, Zhou X, Zheng Y, Zhang W, Zhu JK: Identification of novel and candidate miRNAs in rice by high throughput sequencing. BMC Plant Biol 2008, 8:25.

21. Yao Y, Guo G, Ni Z, Sunkar R, Du J, Zhu JK, Sun Q: Cloning and characterization of microRNAs from wheat (Triticum aestivum L.). Genome biology 2007, 8(6):R96.

22. Friedlander MR, Chen W, Adamidi C, Maaskola J, Einspanier R, Knespel S, Rajewsky N: Discovering microRNAs from deep sequencing data using miRDeep. Nature biotechnology 2008, 26(4):407-415.

23. Megraw M, Baev V, Rusinov V, Jensen ST, Kalantidis K, Hatzigeorgiou AG: MicroRNA promoter element discovery in Arabidopsis. RNA 2006, 12(9):1612-1619.

24. Warthmann N, Das S, Lanz C, Weigel D: Comparative Analysis of the MIR319a MicroRNA Locus in Arabidopsis and Related Brassicaceae. Molecular biology and evolution 2008, 25(5):892-902.

25. Szarzynska B, Sobkowiak L, Pant BD, Balazadeh S, Scheible WR, MuellerRoeber B, Jarmolowski A, Szweykowska-Kulinska Z: Gene structures and processing of Arabidopsis thaliana HYL1-dependent pri-miRNAs. Nucleic Acids Res 2009, 37(9):3083-3093.

26. Jaillon O, Aury JM, Noel B, Policriti A, Clepet C, Casagrande A, Choisne N, Aubourg S, Vitulo N, Jubin C, et al: The grapevine genome sequence suggests ancestral hexaploidization in major angiosperm phyla. Nature 2007, 449(7161):463-467

27. Griffiths-Jones S, Saini HK, van Dongen S, Enright AJ: miRBase: tools for microRNA genomics. Nucl Acids Res 2008, 36(suppl_1):D154-158.

28. Barakat A, Wall K, Leebens-Mack J, Wang YJ, Carlson JE, Depamphilis CW: Large-scale identification of microRNAs from a basal eudicot (Eschscholzia californica) and conservation in flowering plants. Plant Journal 2007, 51:991-1003.

29. Moxon S, Jing R, Szittya G, Schwach F, Rusholme Pilcher RL, Moulton V, Dalmay T: Deep sequencing of tomato short RNAs identifies microRNAs targeting genes involved in fruit ripening. Genome Res 2008, 18:1602-1609.

30. Vaucheret $\mathrm{H}$ : Post-transcriptional small RNA pathways in plants: mechanisms and regulations. Genes \& Development 2006, 20(7):759-771.

31. Meyers BC, Axtell MJ, Bartel B, Bartel DP, Baulcombe D, Bowman JL, Cao X, Carrington JC, Chen X, Green PJ, et al: Criteria for Annotation of Plant MicroRNAs. Plant Cell 2008, 20(12):3186-3190.

32. Dohm JC, Lottaz C, Borodina T, Himmelbauer H: Substantial biases in ultra-short read data sets from high-throughput DNA sequencing. Nucleic Acids Research 2008, 36(16):e105.

33. Barad O, Meiri E, Avniel A, Aharonov R, Barzilai A, Bentwich I, Einav U, Gilad S, Hurban P, Karov Y, et al: MicroRNA expression detected by oligonucleotide microarrays: system establishment and expression profiling in human tissues. Genome Research 2004, 14(12):2486-2494.

34. Li WX, Oono Y, Zhu J, He XJ, Wu JM, lida K, Lu XY, Cui X, Jin H, Zhu JK: The Arabidopsis NFYA5 transcription factor is regulated transcriptionally and posttranscriptionally to promote drought resistance. Plant Cell 2008, 20:2238-2251. 
35. Arazi T, Talmor-Neiman M, Stav R, Riese M, Huijser P, Baulcombe DC Cloning and characterization of micro-RNAs from moss. The Plant Journal 2005, 43(6):837-848.

36. Choi $D, K i m ~ J H$, Kende $H$ : Whole genome analysis of the OsGRF gene family encoding plant-specific putative transcription activators in rice (Oryza sativa L.). Plant \& Cell Physiology 2004, 45(7):897-904.

37. Kim JH, Choi D, Kende $\mathrm{H}$ : The AtGRF family of putative transcription factors is involved in leaf and cotyledon growth in Arabidopsis. Plant Journal 2003, 36(1):94-104.

38. Liu H-H, Tian X, Li Y-J, Wu C-A, Zheng C-C: Microarray-based analysis of stress-regulated microRNAs in Arabidopsis thaliana. RNA 2008, 14(5):836-843.

39. Lauter N, Kampani A, Carlson S, Goebel M, Moose SP: microRNA172 downregulates glossy15 to promote vegetative phase change in maize. Proc Natl Acad Sci USA 2005, 102(26):9412-9417.

40. Terrier N, Glissant D, Grimplet J, Barrieu F, Abbal P, Couture C, Ageorges A Atanassova R, Leon C, Renaudin JP, et al: Isogene specific oligo arrays reveal multifaceted changes in gene expression during grape berry (Vitis vinifera L.) development. Planta 2005, 222(5):832-847.

41. Sunkar R, Zhu J-K: Novel and Stress-Regulated MicroRNAs and Other Small RNAs from Arabidopsis. Plant Cell 2004, 16(8):2001-2019.

42. Sunkar R, Kapoor A, Zhu J-K: Posttranscriptional Induction of Two Cu/Zn Superoxide Dismutase Genes in Arabidopsis Is Mediated by Downregulation of miR398 and Important for Oxidative Stress Tolerance. Plant Cell 2006, 18(8):2051-2065.

43. Abdel-Ghany SE, Pilon M: MicroRNA-mediated systemic down-regulation of copper protein expression in response to low copper availability in Arabidopsis. Journal of Biological Chemistry 2008, 283(23):15932-15945.

44. McCaig BC, Meagher RB, Dean JF: Gene structure and molecular analysis of the laccase-like multicopper oxidase (LMCO) gene family in Arabidopsis thaliana. Planta 2005, 221(5):619-636.

45. Liang M, Davis E, Gardner D, Cai X, Wu Y: Involvement of AtLAC15 in lignin synthesis in seeds and in root elongation of Arabidopsis. Planta 2006, 224(5):1185-1196.

46. Hirsch J, Lefort V, Vankersschaver M, Boualem A, Lucas A, Thermes C, d'Aubenton-Carafa Y, Crespi M: Characterization of 43 non-protein-coding mRNA genes in Arabidopsis, including the MIR162a-derived transcripts. Plant Physiology 2006, 140(4):1192-1204.

47. Rybak A, Fuchs H, Smirnova L, Brandt C, Pohl EE, Nitsch R, Wulczyn FG: A feedback loop comprising lin-28 and let-7 controls pre-let-7 maturation during neural stem-cell commitment. Nature cell biology 2008, 10(8):987-993.

48. Denoeud F, Aury JM, Da Silva C, Noel B, Rogier O, Delledonne M, Morgante M, Valle G, Wincker P, Scarpelli C, et al: Annotating genomes with massive-scale RNA sequencing. Genome biology 2008, 9(12):R175.

49. Shahmuradov IA, Solovyev W, Gammerman AJ: Plant promoter prediction with confidence estimation. Nucleic Acids Res 2005, 33(3):1069-1076.

50. Vaucheret $\mathrm{H}$, Mallory AC, Bartel DP: AGO1 homeostasis entails coexpression of MIR168 and AGO1 and preferential stabilization of miR168 by AGO1. Molecular Cell 2006, 22(1):129-136.

51. Amrani N, Sachs MS, Jacobson A: Early nonsense: mRNA decay solves a translational problem. Nature Reviews Molecular Cell Biology 2006, 7(6):415-425.

52. Rezaian MA, Krake LR: Nucleic acid extraction and virus detection in grapevine. Journal of Virological Methods 1987, 17(3-4):277-285.

53. Barnes WM: PCR amplification of up to $35-\mathrm{kb}$ DNA with high fidelity and high yield from lambda bacteriophage templates. Proceedings of the National Academy of Sciences of the United States of America 1994, 91(6):2216-2220.

54. Carra A, Gambino G, Schubert A: A cetyltrimethylammonium bromidebased method to extract low-molecular-weight RNA from polysaccharide-rich plant tissues. Analytical Biochemistry 2007, 360(2):318-320.

55. Bolstad BM, Irizarry RA, Astrand M, Speed TP: A comparison of normalization methods for high density oligonucleotide array data based on variance and bias. Bioinformatics 2003, 19(2):185-193.

56. Gambino G, Perrone I, Gribaudo I: A Rapid and effective method for RNA extraction from different tissues of grapevine and other woody plants. Phytochem Anal 2008, 19(6):520-525.

57. Li R, Li Y, Kristiansen K, Wang J: SOAP: short oligonucleotide alignment program. Bioinformatics 2008, 24(5):713-714.
58. Wheelan SJ, Church DM, Ostell JM: Spidey: a tool for mRNA-to-genomic alignments. Genome Research 2001, 11(11):1952-1957.

doi:10.1186/1471-2164-11-109

Cite this article as: Mica et al: Correction: High throughput approaches reveal splicing of primary microRNA transcripts and tissue specific expression of mature microRNAs in Vitis vinifera. BMC Genomics 2010 11:109.

\section{Submit your next manuscript to BioMed Central and take full advantage of:}

- Convenient online submission

- Thorough peer review

- No space constraints or color figure charges

- Immediate publication on acceptance

- Inclusion in PubMed, CAS, Scopus and Google Scholar

- Research which is freely available for redistribution
C Biomed Central 